\title{
الإقطاع ونشأة الجيش الصليبي ببيت المقدس
}

محمد علي إسماعيل ؛؛ عبد الله سالم بازينة؛ فاطمة إبراهيم طرينة؛ محمد الحمد البيطة

$$
\text { كلية الآداب - جامعة مصراتة }
$$

تاريخ التقديم: 25-03-2021، تاريخ القبول: 30-05- 2021، نشر إلكترونيا في 02-06-2021

https://doi.org/10.36602/faj/2021.n.17.12

\section{ملخص البحث:}

نجح الصليبيون في تكوين عدة محالك صليبية في المشرق الإسلامي، و تأثرت عاداتما ومؤسساتما بالوافدين من أوروبا الغربية، فقد كان هنالك على الدوام اتصالات وثيقة، غير أها كممالك صغيرة نسيباً غالبا ما افتقرت إلى الدعم المالي والعسكري المتواصل من أوروبا، الأمر الذي جعلها تعمل على رأب ذلك؛ ليصبح النظام الحاكم في هذه الممالك هو النظام الإقطاعي، فقد اعتمدت هذه الممالك في بداية نشأتها على النبلاء والإقطاعيين القادمين من أوروبا، إلا أن معظم هؤلاء يرجعون لموطنهم الأصلي بعد انتهاء دورهم العسكري وتقديم خدماقم، وللمحافظة على الوجود الصلبي في المشرق، منحت المدن التي تمكن الصليبيون من الاستيلاء عليها للسادة الإقطاعيين؛ ليحافظوا عليها في ظل غياب القوة العسكرية والبشرية الفاعلة، ومن هنا بدأت تظهر ملامح تشكل الجيش الإقطاعي الصليبي موضوع الدراسة. الكلمات المفتاحية: الاقطاع، الحروب الصليبية، الجيث الصليبي، الفرسان 


\title{
Feudalism and the Emergence of the Crusader Army in Jerusalem.
}

\author{
Muhammad A. Ismail; Abdullah S. Bazina; Fatima I. Traina \\ Muhammad M. Al-Beeta \\ Faculty of Arts - Misurata University
}

\begin{abstract}
:
The Crusaders succeeded in forming several Crusader kingdoms in the Islamic East، and their customs and institutions were influenced by expatriates from Western Europe، as there were always close contacts * but as relatively small kingdoms، they often lacked financial and military support from Europe، which made them work to remedy that. To become the ruling system in these kingdoms is the feudal system * as these kingdoms relied at the beginning of their inception on the nobles and feudal lords coming from Europe، but most of them return to their original home after the end of their military role and the provision of their services ' and to maintain the Crusader presence in the East، cities were given that could enable The Crusaders from seizing it for the feudal lords; to preserve it in the absence of effective military and human force، and from here the features of the formation of the Crusader feudal army began to appear، the subject of study.
\end{abstract}

Key Words: Feudalism ‘ Crusades ‘ Crusade Army ‘ Knights

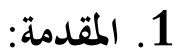

شكل نظام الإقطاع عصب الحياة السياسية والاقتصادية في غرب أوروبا خلال فترة

العصر الوسيط، فقد احدث ظهور النظام الإقطاعي ضعف السلطة المركزية في الغرب

الأوروبي، الأمر الذي أدى لنشوب النزاعات والحروب بين الأمراء الإقطاعيين، حيت لم 
تكن هنالك قوة عليا قوية قادرة على وضع الحد وإيقاف تلك الحروب التي شنها أمراء الإقطاعيات ضد بعضهم، وأحيانا ضد الملك، بذلك حتم الأمر على الكنسية التدخل لإيقاف الحروب المتجددة باستمرار في ظل فقدان الدولة سيادةّا، أمام تنامي السلطة الملكية الفردية، بذلك حازت البابوية قصب السبق في إنهاء الحروب الإقطاعية، والعمل على إحلال السلام في غرب أوروبا، بالدعوة لعقد عدد من المجامع الدينية، صيغت من خلالما عدة قوانين ووضعت المواثيق، كما شكلت عدة هيئات ومنظمات هدفها تنفيذ القرارات الدينية الداعية لإحلال السلام وتطبيق هدنة الله_حسب معتقدهم_ ونشأت هذه الحركة أول أمرها في بلاد الغال (فرنسا)، لتنتشر بعدها لسائر المناطق والبلاد في الغرب الأوروبي.

وبطبيعة الحال عندما أصبحت البابوية على رأس العالم المسيحي الأوروبي في أواخر القرن الحادي عشر الميلادي، أخذت على عاتقها ضرورة المحافظة على السلام واستمراره في أوروبا الغربية، حيت ندد البابا (أربانوس الثاني) في مؤتمر كليرمون 1095م، الذي دعا فيه للقيام بحملات صليبية على المشرق الإسلامي بالحروب الأميرية الإقطاعية، وما جرته من ويلات على الغرب الأوروبي بكافة أطيافه، وعلى مختلف الأصعدة الديمرغرافية والسياسية والاقتصادية والدينية، وأكد على إن هذه الحروب يجب أن توجه لخدمة أوروبا وكنيستها بإعلان الجهاد المقدس للسيطرة على بيت المقدس، والحفاظ على المعالم الدينية المسيحية في المشرق الإسلامي. بذلك وجد أمراء الإقطاعيات في هذه الدعوات فرصة لإشباع رغباقم القتالية، فضلا عن استمرار صراعاهم الحربية على أرض جديدة، بما يضمن لهم تحقيق أطماعهم وتوسيع رقعة أملاكهم الإقطاعية. 
في تسليط الضوء على النظام الإقطاعي في الممالك الصليبية ودوره في نشأة وتكوين الجيش الصليبي، وما يقدمه أصحاب الاقطاعات ومستأجريها من خدمات للفرسان والمشاة على أساس العلاقات الإقطاعية من إقطاعيات الأرض، وإقطاعيات المال. 2.1 - 2.1

تمدف الدراسة الى معرفة كيفية ظهور النظام الإقطاعي في المشرق بعد عبوره إليها، والوقوف عن كثب على دوره في تكوين الجيوش الصليبية في بيت المقدس بمختلف مكوناها، وكافة أطيافها، فضلاً عن معرفة أثره في استمرار الوجود الصليبي في بيت المقدس خاصة، والمشرق الإسلامي بشكل عام. 3.1

تعالج الدراسة دور نظام الإقطاع في تكوين ونشأة الجيش الصليبي، ومدى ارتباطه بنظام الفروسية الذي ساد في أوروبا الغربية؛ لارتباطه بالطبقة الإقطاعية؛ لكونه نبت من بيئتها. وتطرح التساؤلات الآتية:

ما هو الإقطاع؟ وكيف ظهر في الغرب الأوروبي؟ وما علاقته بنظام الفروسية؟ وما الدور الذي لعبه نظامي الإقطاع والفروسية في نشأة الجيش الصليبي في المشرق؟ وكيف عملت الكنسية على أعلاء شأن ومكانة نظام الفروسية؟ ولماذا احتضنته؟ ما أثر تطبيق هذا النظام في المشرق على أوروبا الغربية آنذاك؟ 4.1

لعل الأمراء الإقطاعيين وجدوا في أراضي المشرق الإسلامي أرضاً خصبةً لنمو النظام الإقطاعي، ليزداد إيقاعه بمرور الوقت، ويصبح استمراراً للعصر الإقطاعي الأوروبي، بكل 
مقوماته وحروبه ونزاعاته، الذي بلغ ذروته خلال القرنين الحادي عشر والثاني عشر الميلاديين.

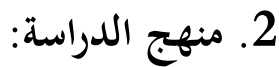

تنتهج هذه الدراسة منهجاً تاريخياً يعتمد على سرد الأحداث التاريخية، بعد بتميع النصوص لدراستها، وتحليلها؛ فضلا عن الاستعانة ببعض المراجع المتعلقة بموضوع الدراسة، بغية الوصول لنتائج علمية وتاريخية واضحة. 3.

\section{3 تعريف الإقطاع (Feudalism):}

كلمة إقطاع مشتقة من كلمة (اقتطاعية) وهي قطعة أرض يشرف عليها أحد النبلاء من أجل الاستفادة منها (نيفين،2011، ص2157)، وقد تعددت آراء الباحثين وتعريفاتم للإقطاع وقد رأيت أن أقتصر بهذا التعريف الدقيق للإقطاع بأنه:"عبارة عن طائفة من النظم فرضت على الرجل الحر الولاء أو التبعية والحندمة، ولاسيما الحربية، يؤديها لرجل حر آخر هو السيد الذي يلتزم بحماية تابعه والإنفاق عليه، وأن ييذل له قطعة أرض

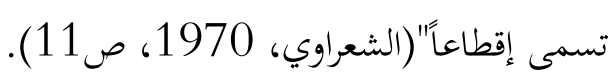

أُطلق مصطلح النظام الإقطاعي على النظام الاقتصادي، والاجتماعي، والسياسي الذي ساد أوروبا خلال العصور الوسطى، والذي ظل سائداً حتى القرن الرابع عشر الميلادي، ويرتبط هذا النظام ارتباطاً وثيقاً بالأرض، فمن يملكها هو السيد والحاكم، وكان أصحاب النفوذ والسلطان في تلك الفترة هم ملاك الأراضي الكبيرة (سيد، 2008، ص (27) 
يرتكز النظام الإقطاعي على ثلاث أسس جوهرية تتمثل في السيد- التابع الإقطاع، حيث يرتبط التابع بالسيد ويقدم له الولاء والخدمة ليحصل نظير تلك الخدمة على إقطاع وعادة ما يكون عبارة عن قطعة أرض (الشعراوي، 1970، ص12) بعد أن استقر الصليبيون في بلاد الشام، وأسسوا مملكة بيت المقدس، اتفقوا على اختيار أحد أمراء الحملة الصليبية الأولى وهو الدوق جودفري أوف بويون(1) (Godfrey) of Bouillon) (أول رمضان 492،هـ/22 يوليو 1099م ليكون حاكماً عليهم، وصار همهم الأكبر يتمحور في الكيفية التي ستساعدهم في توسيع نفوذهم والدفاع عن

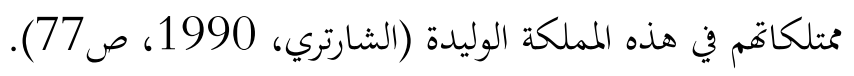
حيث شرع الأمير جودفري في وضع نظام إقطاعي على غرار النظم الإقطاعية التي كانت سائدة في أوروبا أواخر القرن الحادي عشر من أجل الدفاع عن الأراضي التي استولوا عليها وتنظيم الوجود الصليبي فيها (البيشاوي،1989، ص5) فبادر إلى تطبيق الأساليب العسكرية الأوروبية التي خبروها في الغرب الأوروبي، بما يتلائم مع ظروف الشرق ولم يجدوا صعوبة في ذلك، إذ شهدت بلاد الشام تطبيق هذه النظم من قبل السلاجقة الأتراك فبل مجيئهم (عاشور، 2003، ص 2317). بدأت ملامح النظام الإقطاعي الأوروبي ترتسم على أرض الشام منذ الحملة الصليبية الأولى، فهي حملة إقطاعية بامتياز، برزت فيها الأطماع الشخصية للأمراء الصليبين إذ أسسوا إمارتي الرها وأنطاكية ثم بيت المقدس في الفترة من (489-492هـ/1096-

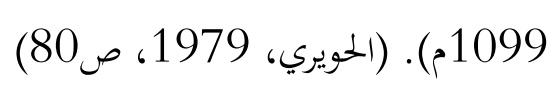

(1) جودفري أوف بويون: ولد عام 1058م، ونشأ في مملكة الفرنجة في إقليم ريمز بمدينة بولونيا، وهو ابن الكونت

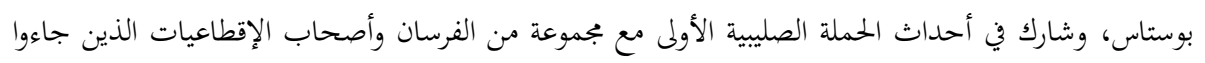

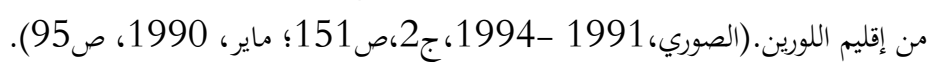


بعد أن وطأت أقدام الصليبين أرض الشام (السرحان،2006، ص2310)، اتخذوا قاعدة لمم في التوزيع الإقطاعي فجعلوا للقادمين الأوائل الحق الكامل في كل حصن أو بلد أو إقليم يضعون أيديهم عليه (طرخان،1968، ص48)، ويؤكد ذلك مؤرخ الحملة الصليبية الأول ريموندا جيل بقوله: "بأن لأول قادم الحق في أن يملك ما استولى عليه من قلعة أو مدينة بشرط أن يرفع علمه عليها". (جيل، 1989، ص235). كان الملك على رأس الهرم الإقطاعي في المملكة (عاشور،2003، ص217)، وتتألف أملاكه من ثلاث مدن كبيرة هي بيت المقدس وعكا ونابلس (1) ثم أضيف إليها الداروم وهي قلعة تقع جنوبي غزة (الشيخ، 2004، ص225)، وكان يلي الملك أربعة من كبار الأمراء، هم أمراء يافا (2) والجليل وصيدا(3) وشرق الأردن.

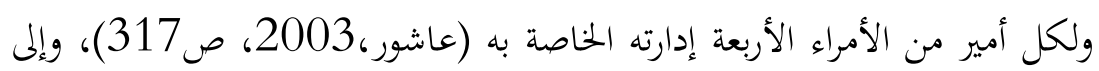
جانب هذه الإقطاعيات الكبيرة، اشتملت المملكة على اثنتي عشرة إقطاعية صغيرة تم منحها إلى أمراء أقل مرتبة لإدارة شؤوها تحت إشراف مباشر لحكومة بيت المقدس (القيتري، 1998، ص62) وكان على كل فصل من هؤلاء الأمراء الكبار والصغار تقديم الولاء والتبعية لسيده الإقطاعي، ويقدم له الخدمة العسكرية والفرسان والمحاربين وفقاً للقواعد

$$
\text { والأصول الإقطاعية (باركر، 1967، صـ57). }
$$

(1) نابلس: مدينة مشهورة بأرض فلسطين تقع بين جبلين مستطيلة لا عرض لها بينها وبين بيت المقدس ثلاثة

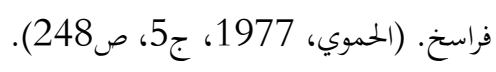

(2) يافا: مدينة فلسطينية تقع على ساحل البحر المتوسط واسمها تحريف لكلمة (يافي) الكنعانية بكعنى جميل وهي

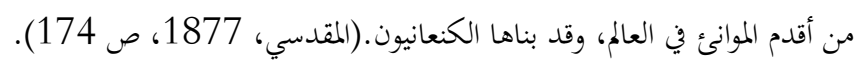

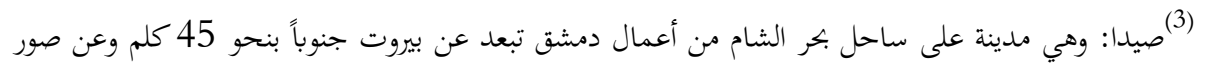

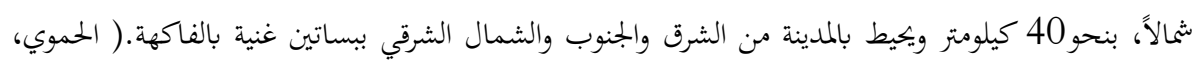

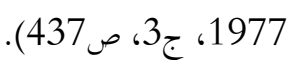


لم تكن الخدمة العسكرية محددة في بلاد الشام بموسم معين أو أيام معدودة كما هو الحال في الغرب الأوروبي، إذ كانت مدة الخدمة محددة بأربعين يوماً، إلا أها في الشرق

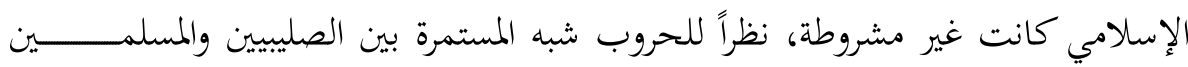

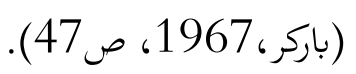

يترأس الملك الهرم الإقطاعي في المملكة الصليبية، وأتباعه من النبلاء على حسب العقد الإقطاعي يهكمون الكونتيـات وهي الوحـدات الرئيسية في المملكة، ويتمتع هؤلاء الكونتيـات بصـلاحيات عسـكرية وماليـة وقضـائية في كونتيـاقم (السـرحان، 2006،

كان من واجبات الملك حماية المملكة من الغارات الخارجية التي قد تتعرض لها، ومن واجباته أيضاً إقامة القلاع والحصون اللازمة؛ لحمايتها لذلك لجأ الملوك الصليبيون الأوائل إلى تشييد العديد من القلاع والحصون في مناطق استراتيجية؛ لتكون بمثابة دروع

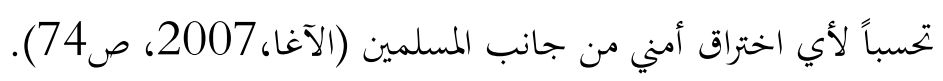
خلاصة القول يمكن أن نصل إلى نتيجة مفادها أن قوة مملكة بيت المقدس قد تكاملت، ورقعتها اتسعت في النصف الأول من القرن الحادي عشر أي خلال عهود ملوكها الأوائل، الذين تميزت فترة حكمهم بالاستقرار الداخلي، إذ عالجوا كثيراً من المشاكل التي واجهتهم عند تأسيس المملكة، وأقاموا نظاماً دفاعياً قوياً وذلك بتشييد العديد من القلاع والحصون لصد هجمات المسلمين. اعتمد ملك بيت المقدس في بناء مملكته بداية على النبلاء، والإقطاعيين القادمين

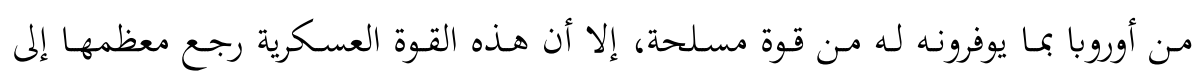

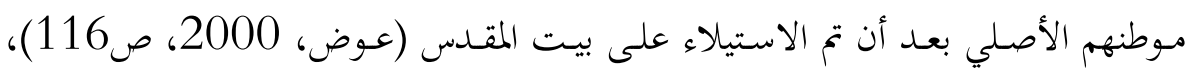
ويعبر عن ذلك المؤرخ فوشيه الشارتري بقوله:"لم يكن هناك من المحاربين ما يكفي للدفاع 
عن المملكة ضد المسلمين إذا ما فكروا في مهاجمتنا..." (الشارتري، 1990، ص166)، ولهذا السبب فإن المـدن التي استطاع الصليبيون الأوائل الاستيلاء عليها مُنحت للسـادة الإقطاعيين لكي يحافظوا عليها في ظل عدم وجـود قوة عسكرية وبشرية فاعلـة خاصـة بالمملكة الصليبية، من هنا بدأت تظهر ملامح تشكيل الجيش الإقطاعي الصلبي، حيث استخدم فيه الصليبيون كافة العناصر المتاحة لمم في تشكيله( محمود،2008، صكئ115). وبالنظر إلى الجيوش الإقطاعية عامة ومن بينها الجيش الإقطاعي الصليبي موضوع الدراسة نلاحظ أها جيوش يتولى قيادتا الأمير أو الدوق أو الماركيز أو الكونت، واعتمدت على الفـارس الذي يلتحق بسـلاح الفرسـان، ويعتبر هـ العمـود الفقـري للجـيش والقـوة الضاربة فيه والمدافع عن حقوق الضعفاء والمظلومين، ولا يمنع ذلك أن يكون هناك فرسان في منتهى الظلم (كاستلان، بدون تاريخ، ص56)، أما الجنود المشاة فيعتبر دورهم ثانوياً إذا مـا قورنوا بالفرسـان، إذكان كل فـارس يمتطي حصـانه يتبعه سائس أو اثنـان مـن المشـاة

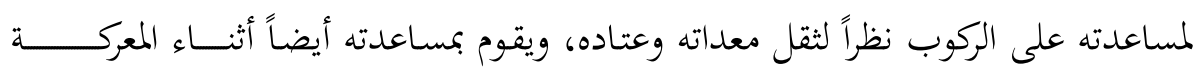
(ماير،1990، ص299؛ قاسم، 1981، 27، ص27) وغالباً ما كان هؤلاء المشاة من الفلاحين الذين يتم تسليحهم بشكل بدائي( كاستلان، بدون تاريخ، ص57). كما كان يسير خلف الجيش الإقطاعي الآلاف من اللقطاء الغوغاء وعادة ماكانوا يسيرون دون قائد أو ضابط، وكانوا يقومون بنهب الأماكن التي فر منها أصحابها أثناء الحرب أو الإجهاز على جرحى العدو بالفؤوس والهراوات كما اتضح ذلك في المشاة الذين

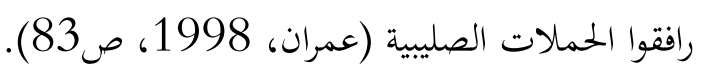

ونظراً لمكانة الفارس في الجيش الإقطاعي الصليبي تبوأ منزلة هامة في المجتمع الصلبي، لفتت أنضار أسامة بن منقذ أحد الشهود العيان لتلك الفترة إذ قال: "والإفرنج خذلهم الله ما فيهم فضيلة من فضائل الناس سوى الشجاعة، ولا عندهم تقدمة ولا منزلة 
عالية إلا الفرسان، ولا عندهم ناس إلا الفرسان، فهم أصحاب الرأي، وهم أصحاب القضاء والحكم..." (ابن منقد، 2003، صنس 135). ولا غرابة في مدى اهتمام الصليبين بالفارس إذ تعتبر الفروسية إحدى سمات العصور الوسطى،وترتبط ارتباطاً وثيقاً بالنظام الإقطاعي وليس من خيار أمام أبناء النبلاء في تلك الفترة إلا الانخراط في سلك الفروسية ليصبحوا فرساناً محاربين، أو الانخراط في سلك رجال الدين نتيجة لهيمة الكنيسة على المجتمع الأوروبي آنذاك (فرح، 2000،

خلاصة القول يمكن أن نصل إلى نتيجة مفادها أن الحروب الصليبية كانت بيئة مناسبة لتطور الفروسية، وعلو شأها في تلك الفترة لأفها استطاعت أن توحد جميع فرسان

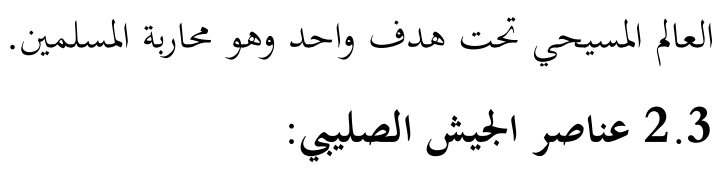
1.2.3 الفرسان (The knights):

تعتبر شريحة الفرسان الركيزة الأساسية لجيش المملكة الصليبية، إذ أن قوة الجيش الصليبي كانت تقاس بعدد الفرسان الذين يضمهم بين صفوفه، بل إن نتيجة المعركة غالباً ما تحسم ضد العدو إذا ما نجح الفرسان في هجومهم (قاسم، 1981، 27، ص23). رغم هذه المكانـة المهمة التي تبوأهـا الفـارس في بنية الجيش الصليبي إلا أن أعـداد هؤلاء الفرسان كانت قليلة في هذا الجيش إذا ما قورنت بأعداد الرجال المشاة وذلك بسبب طبيعة تنشئة الفارس (عمران،1998، ص85) من ناحية وكثرة تكاليف إعداده من ناحية أخرىGaler،1995، (177) حيث أوضح المؤرخ "رانسمان" بأن نسبة الفرسان إلى المشاة في الجيش الصليبي أثنـاء الحملة الصليبية الأولى ربماكانت نسبة واحسد إلى سبعـة 
(رانسمان، 1994، ج1، ص 506) وما يدعم هذا الرأي ما تمدنا به المصادر العربية من أمثلة حيث يذكر ابن القلانسي في حوادث سنة أربعمائة وأربعة وتسعون "أن جيشاً ابحه. من مصر إلى عسقلان لمحاربة الصليبين.... حيث فض له من الفرنج ألف فارس وعشرة آلاف رجل" (ابن القلانسي، بدون تاريخ، ص140) وفي مثال آخر يذكر ابن شداد أن جيش الصليبيين الذي حاصر عكا واستولى عليها عام (588هـ/1191م) كان يتألف من ألفي فارس وثلاثين ألف رجل (ابن شداد، 1994، ص164). كان بتهيز وإعداد هؤلاء الفرسان يتم من قبل الإقطاعيين كخدمة عسكرية لمملكة بيت المقدس، وقد نقلت لنا قوانين بيت المقدس التي دونت في النصف الثاني من القرن السابع الهجري، أعداد الفرسان المطلوب تحصيلها من كل إقطاعية لتشكيل الجيش الصليبي (الآغا،72007، ص75) إذ كان على كل صاحب إقطاعية تقديم عدد من الفرسان للخدمة في الجيش الصلبي، ويصل عدد هؤلاء الفرسان إلى 675 فارس من كل الإقطاعيات الصغيرة والكبيرة بالإضافة إلى فرسان الهيئات الدينية (ماير، 1990، ص (297)

ويتميز هؤلاء الفرسان عن غيرهم بنبل المولد، والبراعة في العمليات القتالية وامتطاء الخيول، وكان يرافق هؤلاء الفرسان خادم أو أكثر يتولون حراسة عتادهم ومعداهم ولا يسهمون في القتال عادة (سميل، 1985، ص 177). كان أصحاب الإقطاعات أو مستأجريها يقدمون خدمات الفرسان على أسـاس العلاقات الإقطاعية، وقد شملت الخدمات الإقطاعية بالإضافة للأرض، إقطاعيات المال، 
وقد ذكر وليم الصوري أن رينولد دي شايتون(1)(Renauld de chatillon) قدم خدماتـه العسـكرية للملـك بلـدوين الثالـث (BaldwinIII) (2) مقابـل إقطساع نقــدي (الصوري، 1994، ج3، ص351) وكان هذا الإقطاع النقدي يجني منه الفارس مبلغاً من المال سنوياً يتراوح بين 400 إلى 600 بيزنت (3). 2.2.3 المشاة (The Sergents)

مصطلح يطلق على بجموعة من الجنود المسلحين بأسلحة خفيفة والمستعدين دائماً للقتال وقد يختلف الاستعداد بين هؤلاء الجنود المشاة من حيث المهارة والتجهيز العسكــري (السرحان، 2006، ص400) فيسمى فرد المشاة الذي لا يحمل سلاح في الجيش الإقطاعي فترة العصور الوسطى ب(الأعزل أو السوقة) (Imbellis) ويصبح مهيأ لقتال العدو ويتلقى تعليماته من قائد المجموعة التي يتبعها حتى يُصبح جندي مشاة ويسمى (بيادة) (Pedites) ( سميل، 1985، صنئ 184)؛ وينقسم المشاة المشاركين في الحملات الصليبية إلى قسمين، قسم مشاة الحجاج المزودين بالسلاح والقادمين إلى الأراضي المقدسة وهؤلاء يشتركون في المعارك بثيابهم المعتادة ويحملون هراوة أو

(1) رينولد دي شايتون: عُرف عند المسلمين باسم أرناوط، وهو فارس خدم في جيش بلدوين الثالث، تزوج من

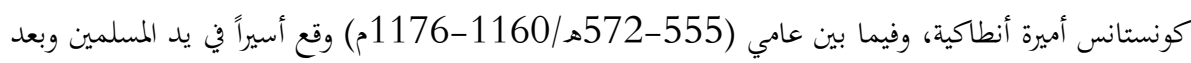

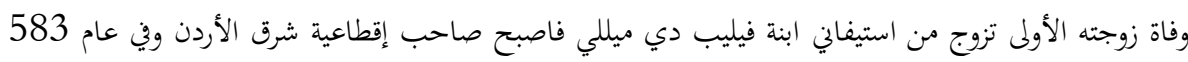

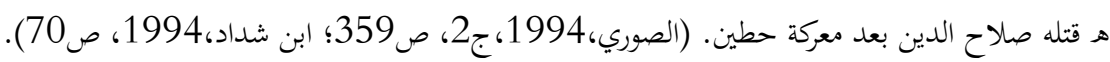

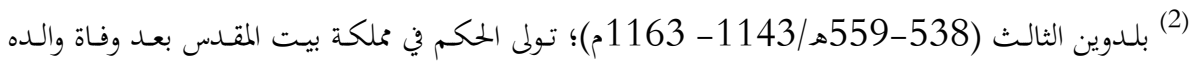

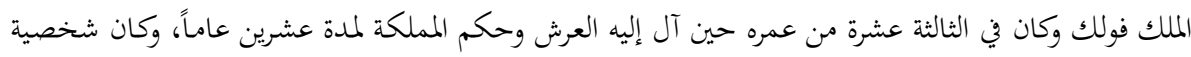

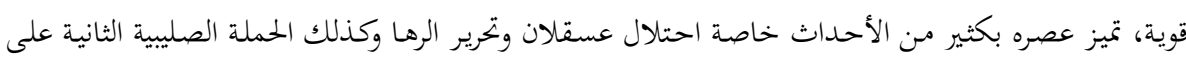

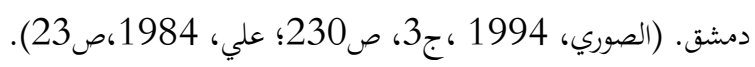

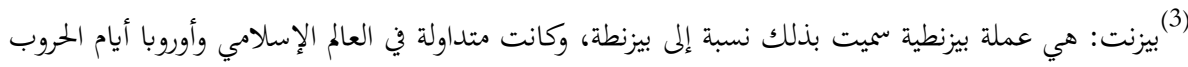

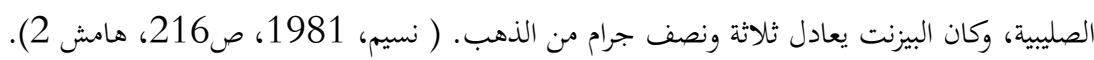


قوساً أو رمحاً (بالار، 2002، ص1402)، وقسم المشاة المحترفين للقتال والذين يرافقون

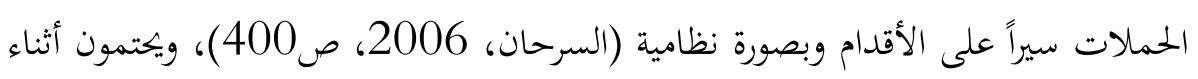
المعارك بدرع أو معطف من الجلد أو الكتان المبطن ويتسلحون برمح أو قوس أو بقاذفة أقواس لقذف السهام والكرات والحجارة، ويشكل هؤلاء المشاة المسلحة ساتراً بين العدو وسلاح الفرسان، ويفتحون له ممراً أثناء إعطاء إشارة الهجوم لهم (بالار،2002، (141)

كانت مصادر بتحنيد هؤلاء الجنود المشاة من ضمن مساعدات الكنيسة والمجتمعات المدنية للدفاع عن المملكة (سميل،1985،ص153)، ففي حالات الطوارئ كان بطريرك

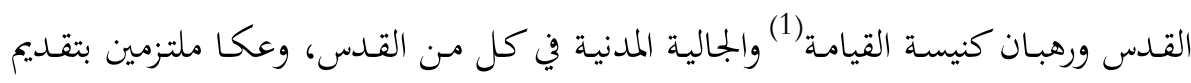
500 جنـــي مشـاة (سـرجندي) (Sergents) (2) عـن كـل جهـة، وأسـاقفة صــور والناصرة(3) وأسقف عكا ورئيس دير جبل صهيون(4) $150^{(4)}$ جندي عن كل مسنهم ... وهكذا بحيـث يكون مجموع الحدمـة المطلوبـ 5025 جندي مشـاة (سرجندي) ( ماير، 1990، ص297) ولم تكـن مـا تقدمـه الكنيسـة متمثلـة في رجـال الدين وكـذلك الطبقـة

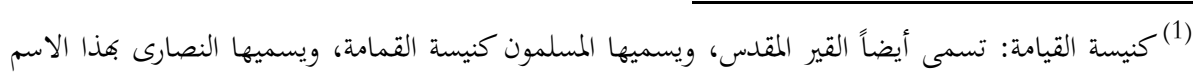

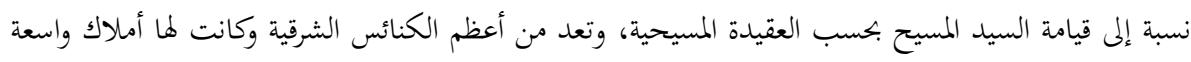

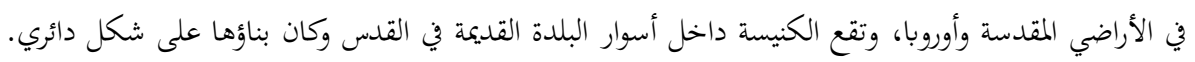

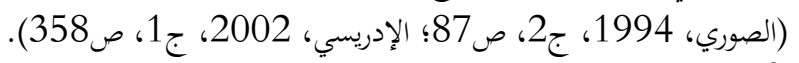

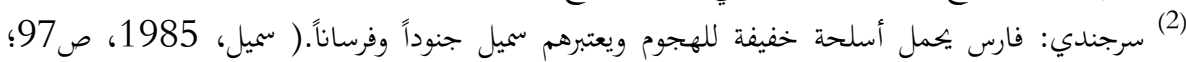

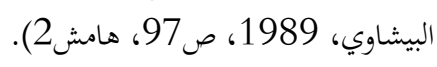

(3) الناصرة: إحدى المدن الفلسطينية وتقع على بعد 197 مالثش 112كلم شثمال بيت المقدس وعلى بعد 75 كلم شمال

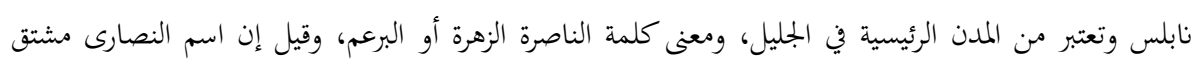

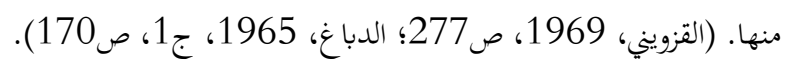
(4) جبل صهيون: أحد الجبال في مدينة القدس ويتبع شرقي المدينة، وأقيمت عليه كنيسة تحمل نفي نس الاسم.

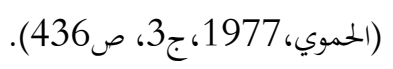


البرجوازية(1) من جنود مشاة للجيش الصلبي، مبنياً على التبعية الإقطاعية، كما هو الحال في طبقة النبلاء والتي تتبع الملك، وإنما كان على أساس أفهم جزء مـ التركيبة السكانية للمملكة وتتأثر متلكاقم عندما تتعرض المملكة لمخاطر (السرحان، 2006، صب401). أمسا مـن حيث دور هؤلاء الجنود المشـاة بتحاه المملكة الصليبية لم يقتصر على المشـاركة في المعارك الحربية ضد المسلمين فحسب، وإنما كان لهم دور إيجابي في حفظ الأمس داخل المملكة (الآغا، 2007، ص77).

\subsection{3 المرتزقة (The Mercenary):}

عانى الصليبيون من مشكلة نقص العنصر البشري طيلة بقائهم في بلاد الشام كثيراً، وترتب على ذلك عجز بعض أمراء الإقطاعيات عن توفير الأفراد المطلوب منهم لدعم جيش المملكة في حال تعرض أتباعهم من الفرسان إمـا لمرض أو غيره، وبالتالي لجأوا إلى توظيف مرتزقة في خدمتهم (السرحان، 2006، ص401) وعادة ما كان هؤلاء المرتزقة من أهل البلاد الأصليين من السريان(2) والموارنة(3) الذين كان منهم رماة النشاب، والأرمـ (1) الذين كانوا من أفضل جنود المشاة في الجيش الصليبي (الشامي، 1985، صنوسين

(1) البرجوازية: هم طبقة الأحرار في مملكة بيت المقدس، ويتتمون للعامة، وقد احتشدت تلك الطبقة في المدن

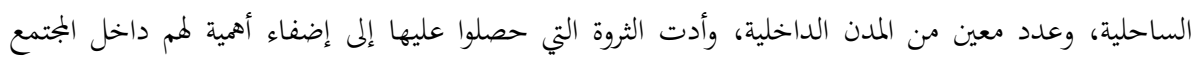

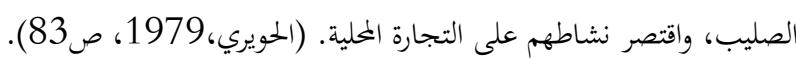

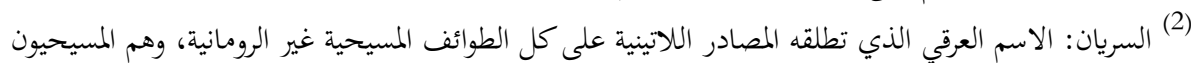

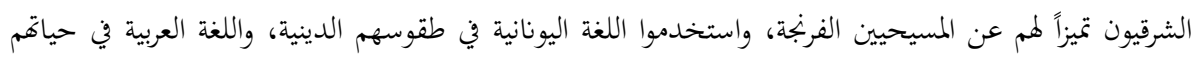

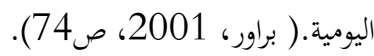

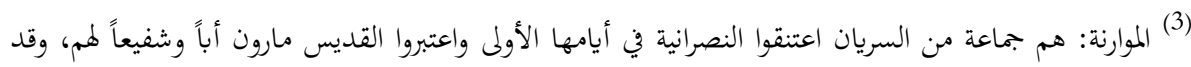

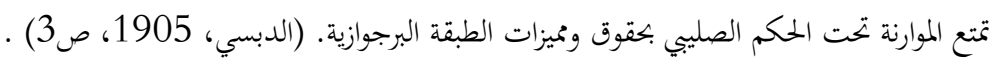


يرجع استخدام المرتزقة في الجيش الصليبي إلى الحملة الصليبية الأولى وقد استمر خلال القرنين الثاني عشر والثالث عشر( بالار، 2002، ص 143) وأحياناً يكون توظيف هؤلاء المرتزقة من الصليبيين القادمين للحج إلى الأراضي المقدسة، ففي سنة (548هـ/1538) أثناء حصار عسقلان وعد الملك بلدوين الثالث الحجاج الذين التحقوا به بإعطائهم أجرة مالية نظير خدماقم (الصوري، 1994، ج3، ص351). وبما أن الصليبيين كانوا بحاجة دائمة إلى استنفار جميع القادرين على حمل السلاح للدفاع عن المملكة الصليبية، كان هناك قوات الاحتياط أو ما يعرف بالتعبئة العامة تلجأ إليها المملكة في أوقات الضرورة، حيث كان يتم استدعاء الفرسان المرتزقة بناءً على طلب الملك الصليبي وفي الغالب يكون هؤلاء المرتزقة ممن يستطيعون استخدام الخيول ( السرحان، 2006، ص202)

وأكثر فرق المرتزقة أهمية فرقة التركبولية (Turcoples) وهم فرقة من الفرسان المدرعة بالدروع الخفيفة وتلي في الأهمية فرقة الفرسان (العناني، 2012، ص151) وكان يتم بتحيدهم في العادة من أهل البلاد الأصليين من السريان والمسلمين (باركر،1967، ص151) ومصطلح تركبولي كان يطلقه البيزنطيون على إحدى فرق جيشهم ويبدوا أن البيزنطيين كونوا هذه الفرقة تشبهاً بالأتراك السلاجقة بعد اهزامهم في معركة ملاذ كرد، ثم نقلها عنهم الصليبيون أثناء مرورهم بالأراضي البيزنطية إبان الحملة الصليبة الأولى(ابن واصل، 1957، ص149، هامش1) وكان الجنود التركبولية رجالاً راكبين، ويطبقون

(1) الأرمن: بعد الامتداد السلجوقي إلى قلقيلية في جنوب شرق آسيا الصغرى نزح الأرمن بأعداد كبيرة وانتشروا في

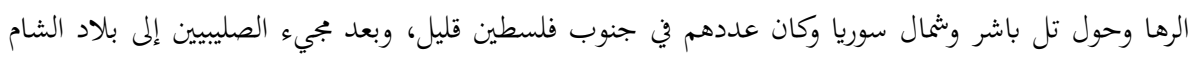

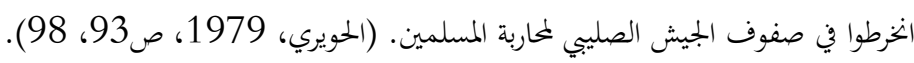


الطرائق التركية في استخدام النبالة الراكبة والقوس ويقومون عادة بمهام الاستطلاع (ابن

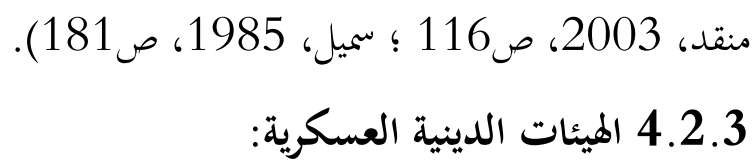

وتشمل هيئة الداوية(1) والاسبتارية(2) وهيئة فرسان التيتون، وهي هيئات تأسست في

الأصل لتلبية احتياجات الحجيج في الأراضي المقدسة، ثم تحولت خلال القرن الثاني عشر

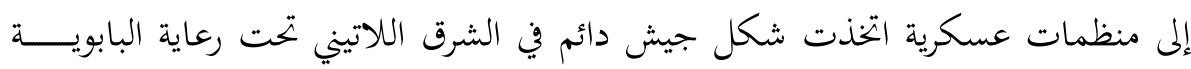
(سميل، 1985، ص159) نتيجة لعدم توفر القوة البشرية الكافية من قبل الطبقة الحاكمة

$$
\text { (السرحان، 2006، صـ5م، (405). }
$$

وكان لعسكرة الهيئات الدينية دور مؤثر في تثبيت الوجود الصليبي في الشرق الإسلامي باعتبارهم قوة مدافعة عن حدود المملكة لأفم سادة القلاع الواقعة على حدود المملكة، وهم المسؤولين عن تزويد هذه القلاع بالجنود والمؤن، حيث تم منح أو بيع هذه القلاع من قبل الحكام والنبلاء إلى هذه الهيئات الدينية لعدم قدرقم على توفير القوة البشرية أو الموارد اللازمة للدفاع عنها ففي عام (575هـ/1180م) أصبحت هيئة الاستبارية مسؤولة بالدفاع عن حوالي خمسة وعشرين قلعة في الشرق، وكان الداوية مسؤولين عن قلعة غزة قرب الحمدود الجنوبية لمملكة بيت المقدس (سميث، 2009، ج2، ص 19).

(1) هم فرسان الصليب الأممر أو فرسان المعبد، وقد تم تأسيس طائفتهم سنة 1118م ، وكان مقرهم الرئيسي في التمان

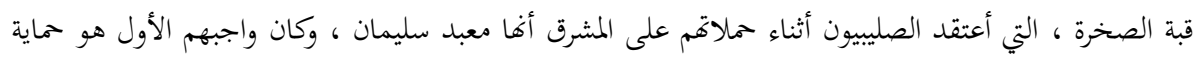

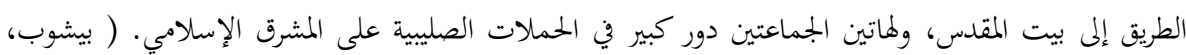

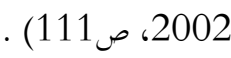
(2) ويرجع تأسيسها إلى عام 111) 1070م، كجماعة متطوعة للعناية بالمرضى والحجاج الفقراء ومساعدقم، ثم دخلوا

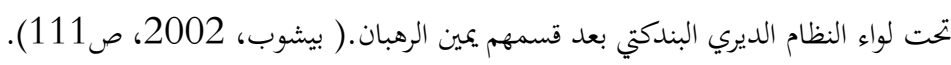


بالإضافة إلى جيش مملكة بيت المقدس بعناصره المختلفة تعتبر أوروبا مصدراً للموارد البشرية العسكرية بما ترسله من جيوش أو ما يفد من حجاج إلى المملكة فبعضهم يلتحق بالجيش والبعض يساهم في تمويله (سميل، 1985، ص 158). 3.3 المساندة البحرية للأساطيل الأوروبية:

لقـد أدرك ملوك المملكة الصليبية الأوائل، مـدى أهمية أن تكـون مملكتهم الناشئة مرتبطة بالبحر، إذ كان بمثابة الشريان الذي يربطهم بالغرب الأوروبي (السرحان، 2006، ص464) لذلك عمل بلدوين الأول على ضم شواطئ وسواحل الشام المواجهة للمملكة الصليبية، والتي كانت تحت سيطرة المسلمين آنذاك وذلك من أجل إقامة حلقة وصل بين المملكة الصليبية والبحر والمدن والموانئ الساحلية، حتى يتسنى تسهيل وصول الأساطيل من غرب أوروبا التي تحمل المؤن والجنود لتقديم الدعم للمملكة (نوار،1992، صنسئ231). ويبدو أن المملكة الصليبية طيلة وجودها في المشرق الإسلامي لم يكن لديها أساطيل خاصة بها، باستثناء بعض السفن الحربية الصغيرة والتي لا تستطيع أن تقارع الأساطيل الفاطمية آنذاك، فأثناء حصار الصليبين لمدينة عسقلان عام (548هـ/1153م) لاذت هذه السفن بالفرار ورُفِع الحصار عن المدينة بمجرد أن اقترب الأسطول الفاطمي منها

$$
\text { (الصوري، 1994، ج3، صن358) }
$$

وقد أشار المؤرخ وليم الصوري لهذه السفن الحربية الصليبية المرافقة للملك عموري

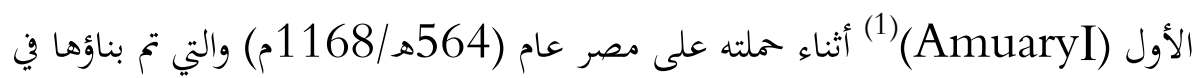

$$
\text { ميناء عكا (الصوري، 1994، ج4، صول (70). }
$$

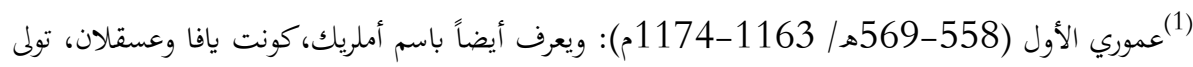

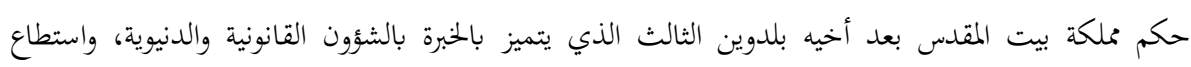


يلجأ الصليبيون كثيراً إلى الاستعانة بالأساطيل الأوروبية في بعض عملياهم العسكرية بسبب ضعف قوتم البحرية، ففي عام (504هـ/1109م) تمكن الصليبيون من الاستياء على مدينة صيدا بمساعدة أسطول سيجورد النرويجي(Sigurd)(1). لم يستعن الصليبيون بالبحرية البيزنطية تلك الفترة لعدة أسباب من أهمها أن البحرية البيزنطية ليس لديها المقدرة على مقارعة البحرية الفاطمية حيث منيت بهزائم أمامها في بداية عهد الخلافة الفاطمية في مصر ولعل السبب الآخر أن البحرية البيزنطية لم تكن مستعدة لمساعدة الصليبين في غزو مدن الساحل إلا إذا تنازل لهم الصليبيون عن أملاكهم السابقة بشمال الشام خصوصاً أنطاكية واللاذقية(2) ولذلك لم يكن أمام الصليبين إلا الاستعانة بمدن إيطاليا التجارية، التي بدأت بتقديم الدعم الرسمي للمملكة الصليبية منذ أن أيقنت بنجاح الحركة الصليبية مقابل امتيازات خاصة يمنحها إياها ملوك هذه الدول (السرجاني،2009، ص363) حيث كانت تشترط هذه المدن عند استخدام سفنها في الحملات الصليبة أن يكون لما أحياء كاملة في الأراضي التي يتم الاستيلاء عليها (عمران، 1998،ص55) كما تمتعت كل مدينة من هذه المدن التجارية بسلطة قضائية وحصانات إدارية، مما جعل هذه الأحياء بلداناً مستقلة ذات حكم ذاتي وأصبحت كل منها مستعمرة

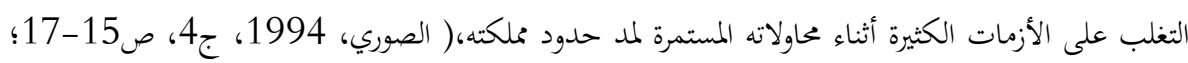

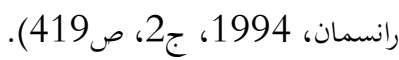

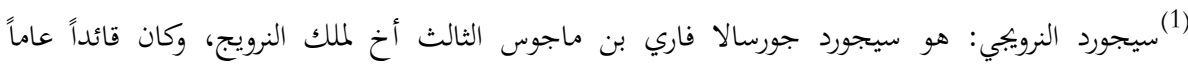

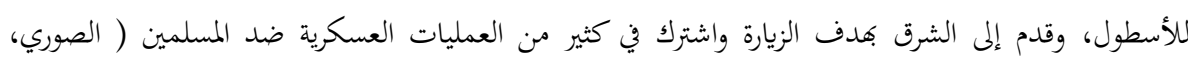

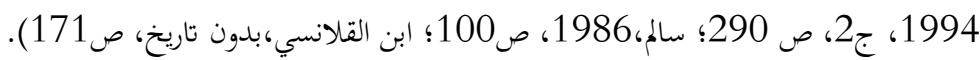

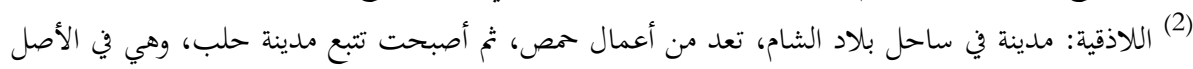

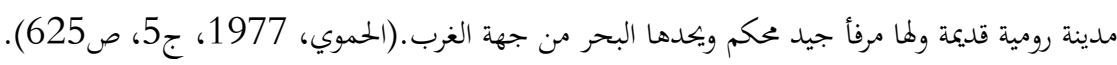


داخل مستعمرة تدار بقائم أعمال قادم من الوطن الأم، يحمل لقب فيكونت (Viconte) استطاع الصليبيون بالمساندة البحرية لهذه المدن التجارية في الفترة من العام (493504هـ/1099-1110م) من السيطرة على جميع مدن الساحل ماعدا صور وعسقلان فأصبحت تلك المدن قواعد سهلت اتصال الصليبين بغرب أوروبا، مما ساعد على وصول الإمدادات البشرية والتموينية لدعم المملكة الصليبية الناشئة كما اتخذ الصليبيون مدينة عكا قاعدة لبناء الآلات الحربية حتى يتمكنوا من حصار مدينتي صور وعسقلان (مكسيموس،

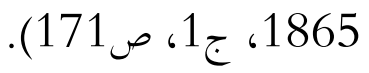
تعتبر جنوة(1) أول المدن الإيطالية التي قامت بتقديم المساعدات العسكرية للصليبين (الصوري، 1994، ج1، ص316) مقابل امتيازات بحارية تحصلت عليها فقد أعطاهم بوهيموند سوقاً خاصاً بهم وكنيسة، كما منحهم ثلاثين بيتاً من بيوت أنطاكية شكلت حياً لنشاطهم التجاري (الصوري، 1994، ج1، ص217؛ عاشور، 2010، ج1، (178) أما مدينة البندقية(2) فهي أكثر المدن التجارية الإيطالية التي استفادت من سيطرة الصليبين على بلاد الشام، حيث تحصلوا على الثلث في كل مدينة يساعدون في الاستيلاء

(1) جنوة: مدينة إيطالية ساحلية تقع في إقليم لغوريا شمال إيطاليا، وهي ميناء بحري هام منذ عهد الجمهورية

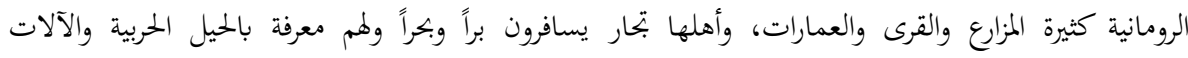

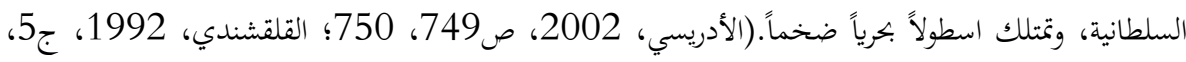
ص406).

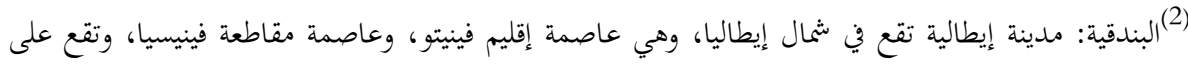

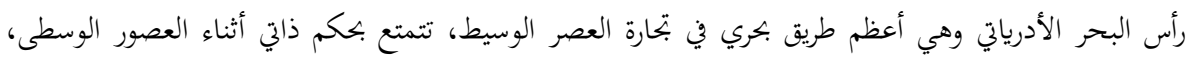


عليها ليقيموا فيها مؤسساتم الخدمية كالفنادق والمخابز والطواحين والحمامات على أن تكون هذه المرافق معفاة من الضرائب والمكوس (الصوري، 1994، ج1، ص379؛ رانسمان، 1994، ج2، ص 199). أما مدينة بيزا(1) الإيطالية، بعد أن قدمت المساعدات للصليبين استطاعت الحصول على امتيازات بتارية، منها شارع في أنطاكية ويي اللاذقية، إلى جانب حرية التجارة في جميع موانئ أنطاكية (عاشور، 2010، ج1، ص 333). كان للمساندة البحرية من قبل أساطيل الغرب الأوروبي أثث إيجابي في حماية الوجود الصلبي وتثبيته في الشرق، ففي عام (517هـ/1123م) حاول المسلمون استغلال أسر الملك بلدوين الثاني (Baldwin II) واستعادة مدينة يافا فنمت محاصرتا براً وبحراً، وتصدى لهم الأسطول البندقي وأحبط المحاولة الإسلامية في استرداد هذه المدينة (الشارتري، 1990، ص256)، وفي أواخر القرن السادس الهجري/الثاني عشر الميلادي اتضح الدور الإيجابي للمدن التجارية في تقديم المساندة البحرية لإعادة احتلال المدن الساحلية التي

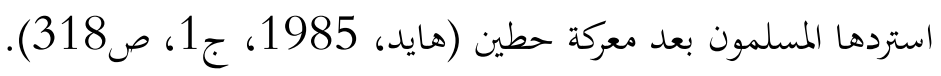

وهي قريبة من جنوة من ناحية البر بينهما نخو ثمانية أيام، وتعتبر أهم مرافئ أوروبا أثناء الحملات الصليبة.

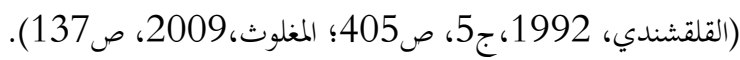

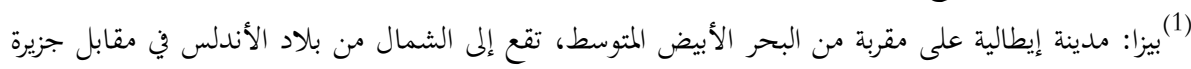

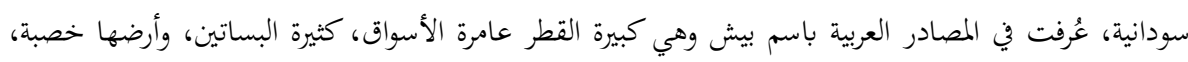

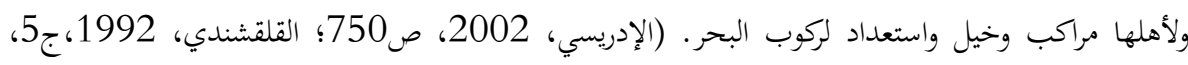

(2) بلدوين الثاني (494-512هـ/1110-1118م): هو ابن هيج كونت رمثيل، وابن عم جودفري وبلدوين

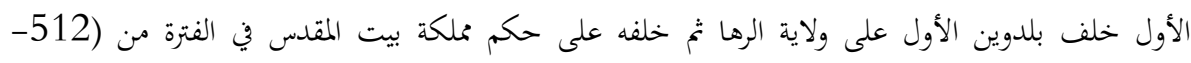

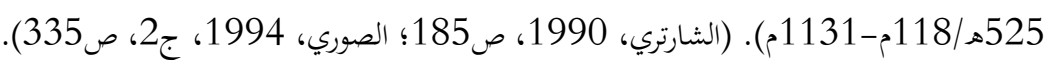


وختاماً نلاحظ أن المدن الإيطالية كانت مصالحها التجارية فوق كل اعتبار من خلال تقديم

$$
\begin{aligned}
& \text { الجانب الاقتصادي على حساب الجانب الديني. }
\end{aligned}
$$

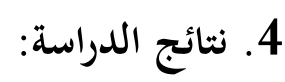

$$
\begin{aligned}
& \text { توصلت الدراسة للنتائج التالية: }
\end{aligned}
$$

- وضحت الدراسة أن ظهور النظام الإقطاعي في المشرق الإسلامي يعد امتداداً لنظام الإقطاع السائد في غرب أوروبا آنذاك؛ ليكون مرآة عاكسة لصور نابضة بالحياة لما تعانيه أوروبا من ضعف السلطة المركزية فيها، فضلاً عن هيمنة أمراء الإقطاعيات على السلطة وكثرة الصراعات الدائرة بينهم؛ الأمر الذي ممكن البابوية من استغلال الوضع لصالحها، بتوظيف هذه العداوات وتوجيهها خدمة لمصالحها الدينية والاقتصادية. - بينت الدراسة أن الحملات الصليبية على المشرق الإسلامي خلقت بيئة مناسبة لتطور نظام الفروسية، الذي ظهر وترعرع في أوروبا الغربية في ظل النظام الإقطاعي، بذلك شكل هؤلاء الفرسان طبقة اجتماعية متميزة لها خصائصها وقوانينها، وخاصة عندما احتضنتهم الكنيسةوأقامت طقوساً وشعائر؛ لتعميدهم ومنحطم رتبة الفروسية لانخراطهم في خدمة البابوية ومصالحها. - أظهرت الدراسة أن تشكل الإقطاعية في الغرب الأوروبي ومن تَّم عبورها للمشرق الإسلامي حدث بتأثير عدة قوى، منحتها حيوية جديدة وقدرة على الاستمرار، وهذه القوى تمثل اندفاع النزعة الاقتصادية الساعية وراء مصالحها وامتيازاتا، والأخرى تنافس دول غرب أوروبا على الزعامة والسيادة في حوض المتوسط، لتلعب المدن الايطالية الدور الأكبر في مساندة الجيش الصليبي في محلكة بيت المقدس تحقيقاً لنزعتها السيادية، فضلاً عن إطماعها الاقتصادية. 
- أن الجيش الصليبي المتواجد في بيت المقدس اعتمد في تكوينه على أمراء الإقطاعيات، وما قدموه هم وإقطاعياقم من دعم ومساندة للجيش بمختلف أطيافه من فرسان ومشاه ومرتزقة؛ ليكون تواجده وحروبه استمراراً لحروبكم الإقطاعية الدائر رحاها في أوروبا في السابق، بشكل لا يخرق المواثيق والقوانين التي وضعتها الكنيسة لحفظ السلام في غرب أوروبا، وكذلك لضمان بقاء الوجود الصليبي في المشرق الإسلامي وخاصة في مملكة بيت المقدس.

\section{المراجع}

أولاً: المراجع العربية:

الإدريسي، أبو عبـدالله محمـد بـن محمـد بـن عبـدالله (2002). نزهـة المشتاق في اختراق الآفاق. القاهرة: مكتبة الثقافة الدينية.

الآغا، حسام علي (2007). الأوضاع الاجتماعية في فلسطين زمن الحرب الصليبية. (رسالة ماجستير غير منشورة). الجامعة الإسلامية، غزة، فلسطين.

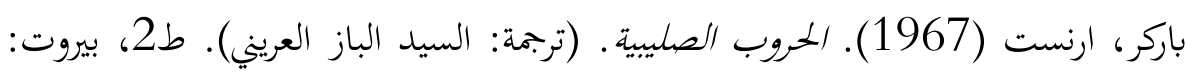
دار النهضة العربية.

بالار، ميشيل(2002). الحملات الصليبية والشرق اللاتيني. (تربمة: بشير السباعي). القاهرة: عين للدراسات والبحوث الإنسانية والاجتماعية.

براور (2001). الاستيطان الصليبي في فلسطين (مملكة بيت المقدس). (ترجمة: عبدالحافظ عبد الخالق البنا). القاهرة: عين للدراسات والبحوث. البيشاوي، سعيد (1989)، الممتلكات الكنيسية في مملكة بيت المقدس، الإسكندرية، دار المعرفة الجامعية. بيشوب، موريس (2002). حضارة أوروبا في العصور الوسطى. (ترجمة: قاسم عبده).

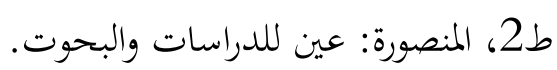


جيل، ريموندا (1989)، تاريخ الفرنجة غزاة بيت المقلس، ترجمة: حسين محمد عطية، الإسكندرية، دار المعرفة الجامعية.

الحموي، شهاب الدين ياقوت بن عبدالله (1977)، معجم البلدان، بيروت، دار صادر.

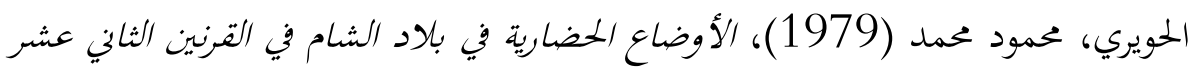
والثالث عشر، القاهرة،دار المعارف.

الدباغ، مصطفى مراد (1965)، بلادنا فلسطين، ج1، قـ1 1، بيروت، دار الهدى.

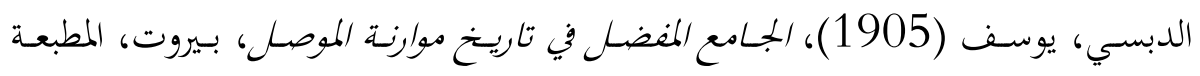
العمومية الكاثوليكية. رانسمان، ستيفن (1994)، تاريخ الحملات الصليبية، ترجمة: نورالدين خليل، ط2، القاهرة، الهيئة المصرية للكتاب.

السرحان، موضى بنت عبدالله (2006)، تنظيمات الصليبين في مملكة بيت المقلس وأثرها على أوضاعهم في بلاد الشام، الرياض، مكتبة الملك فهد الوطنية أثناء النشر.

السرجاني، راغب (2009)، قصة الحروب الصليبية، ط2، القاهرة، مؤسسة اقرأ للنشر. سميت، جونثان ريلي (2009)، تاريخ الحروب الصليبية، ترجمة قاسم عبده، القاهرة، المركز

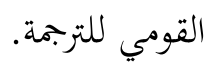

سميل، ر، سي (1985)، فن الحرب عند الصليبين في القرن الثاني عشر، ترجمة: محمد الجلاد، دمشق، مركز الدراسات العسكرية.

سيد، أشرف صالح (2008)، قراءات في تاريخ أوروبا في العصور الوسطى، لبنان، شركة الكتاب العربي الإلكتروني.

الشارتري، فوشيه (1990)، تاريخ الحملة إلى القلس، ترجمة: زياد العسلي، عمان، دار الشروق.

الشامي، أحمد (1985)، العلاقات بين الشرق والغرب في العصور الوسطى، القاهرة، دار النهضة العربية. 
ابن شداد، بهاء الدين (1994)، النوادر السلطانية ومحاسن اليوسفية، تحقيق: جمال الدين الشيال، القاهرة، مكتبة الخانجي للنشر.

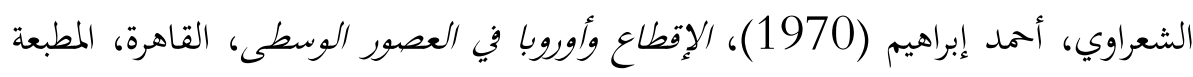
العالمية.

$$
\text { الشيخ، محمد مرسي (2004)، عصر الحروب الصليبية في الشرق، (د.ن)، (د.م). }
$$

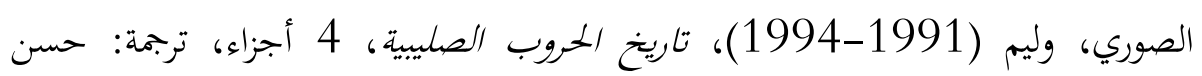
حبشي، القاهرة، الهيئة المصرية للكتاب.

طرخان، إبراهيم (1968)، النظم الإقطاعية في الشرق الأوسط في العصور الوسطى، القاهرة، الكتاب العربي للطباعة.

عاشور، سعيد عبدالفتاح (2003)، تاريخ العلاقات بين الشرق والغرب، ط2، بيروت، دار النهضة العربية.

على، عبد الحفيظ محمد (1984)، مشكلات الوراثة في مملكة بيت المقدس وأثرها على تاريخ الحركة الصليبية، القاهرة، دار النهضة العربية.

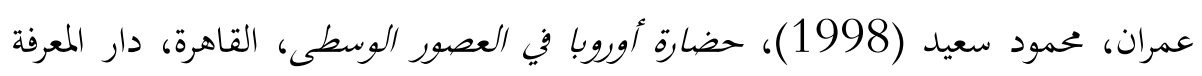
الجامعية.

العناني، جاسر على (2012)، فتح صلاح الدين الأيوبي لبيت المقدس بين السياسة والحرب،، ط1، عمان، أمواج للنشر والتوزيع.

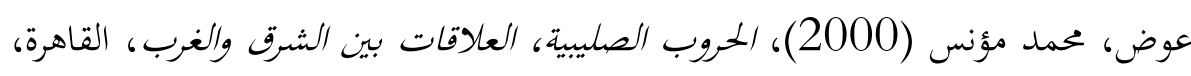
عين للدراسات والبحوث الإنسانية والاجتماعية.

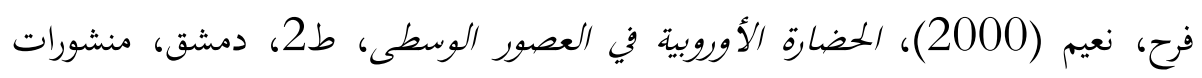
جامعة دمشق.

قاسم، قاسم عبده (1981)، صورة المقاتل الصليبي في المصادر العربيـة، الملجلة التاريخية

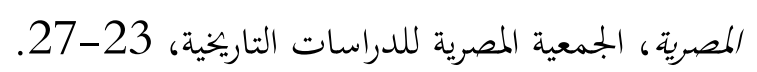


القزويني، زكريا بـن محمــ بـن محمـود (1969)، آثار السباد وأخبار العباد، بيروت، دار صادر.

ابن القلانسي، أبو يعلى حمزة (د.ت)، ذيل تاريخ دمشق، القاهرة، مطبعة المتبي.

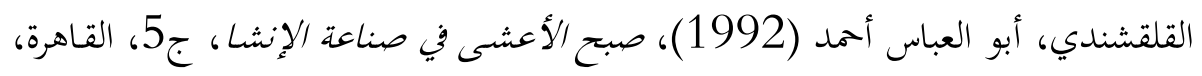
دار الكتب الخديوية.

القيتري، يعقوب (1998)، تاريخ بيت المقدس، ترجمة: سعيد البيشاوي، عمان، دار الشروق.

كاستلان، جورج ألفا (بدون تاريخ)، تاريخ الجيوش، ترجمة: كمال دسوقي (د.س)، (د.م). ماير، هانز ابرهارد (1990)، تاريخ الحروب الصليبية، ترجمة: عماد الدين غانم، طرابلس، منشورات جامعة الفاتح.

$$
\text { محمد مرسي (2004)، عصر الحروب الصليبية في الشرق، (م.ن)، ( د.م). }
$$

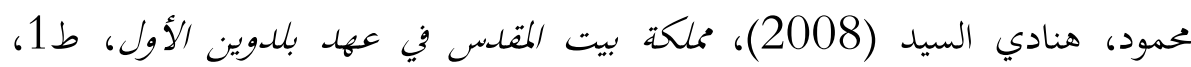

$$
\text { القاهرة، دار العالم العربي. }
$$

المغلوث، سامي عبدالله (2009)، أطلس الحملات الصليبية على المشرق الإسلامي في العصور الوسطى، ط1، الرياض فهرسة مكتبة الملك فهد أثناء النشر.

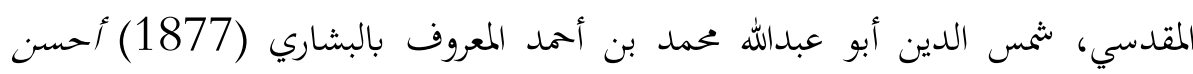
التقاسم في معرفة الأقاليم، لندن، مطبعة بريل.

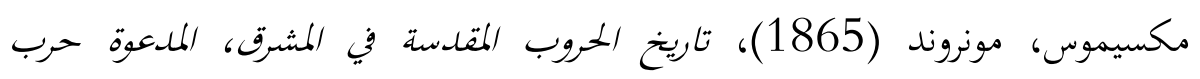

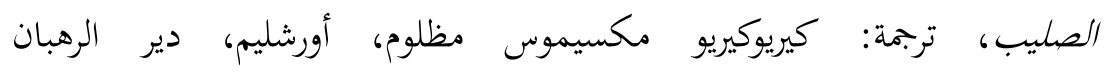
الفرنسيسكان.

ابن منقد، أسامة الكناني الشيزري (2003)، كتاب الاعتبار، تحقيق: عبدالكريم الأشتر، ط2، بيروت، المكتب الإسلامي. 
نسيم، جوزيف (1981)، العلوان الصليبي على مصر (هنيمة لويس التاسع في المنصورة)، طم، بيروت.

نوار، صلاح الدين محمد (1992)، العدوان الصليبي على العالم الإسلامي، ط1، الإسكندرية، دار الدعوة للطباعة والنشر.

نيفين (2011)، الأوضاع الدينية والسياسية والاقتصادية والاجتماعية في الغرب الأوروبي من القرن التاسع حتى القرن الحادي عشر، رسالة ماجستير غير منشورة، الجامعة الإسلامية، غزة، فلسطين.

هايد، فيليام (1985)، تاريخ التجارة في الشرق الأدنى في العصور الوسطى، ترجمة: أحمد

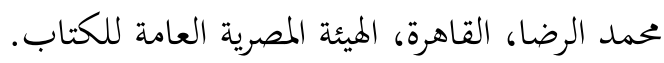

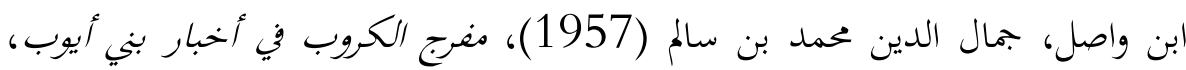
تحقيق: جمال الدين الشيال، القاهرة، المطبعة الأميرية.

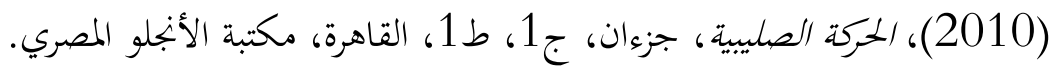

ثانيا: المراجع الأجنبية: Claude, Galler (1995). La valeur militaire des armees De La premiére croisa. Fayaird، Paris. 